\title{
Prédication et liturgie textuelle dans Leche de la fe del príncipe christiano (1545) de Luis de Maluenda
}

Predicazione e liturgia testuale in Leche de la fe del príncipe

christiano (1545) di Luis de Maluenda

Preaching and Textual Liturgy in Luis de Maluenda's Leche de la fe del

príncipe christiano (1545)

\section{Fabrice Quero}

\section{OpenEdition \\ Journals}

Édition électronique

URL : http://journals.openedition.org/cei/6341

DOI : $10.4000 /$ cei.6341

ISSN : 2260-779X

Éditeur

UGA Éditions/Université Grenoble Alpes

Édition imprimée

ISBN : 978-2-37747-159-1

ISSN : $1770-9571$

Référence électronique

Fabrice Quero, « Prédication et liturgie textuelle dans Leche de la fe del príncipe christiano (1545) de Luis de Maluenda », Cahiers d'études italiennes [En ligne], 29 | 2019, mis en ligne le 30 septembre 2019, consulté le 28 mars 2021. URL : http://journals.openedition.org/cei/6341 ; DOI : https://doi.org/ 10.4000/cei.6341

Ce document a été généré automatiquement le 28 mars 2021.

(c) ELLUG 


\title{
Prédication et liturgie textuelle dans Leche de la fe del príncipe christiano (1545) de Luis de Maluenda
}

\author{
Predicazione e liturgia testuale in Leche de la fe del príncipe \\ christiano (1545) di Luis de Maluenda \\ Preaching and Textual Liturgy in Luis de Maluenda's Leche de la fe del \\ príncipe christiano (1545)
}

Fabrice Quero

Prendre le sermon pour objet d'étude, c'est interroger un objet altéré, pour ne pas dire dénaturé, à jamais inconnaissable dans sa forme première et ultime à la fois ; c'est accepter d'ignorer la dimension performative et esthétique première de ce qui est discours et acte de langage ${ }^{1}$, pour mieux se lancer dans une archéologie des contingences qui permettent de s'en approcher. Mais dans une telle entreprise, il n'est pas que les circonstances qui fassent défaut. En amont, tout ce qui intéresse la formation littéraire des orateurs sacrés, et qui conditionne la physionomie littéraire de ce qui nous est parvenu sous forme écrite, ne nous est guère accessible, même à travers les prescriptions répétitives des artes concionandi ${ }^{2}$. À cet égard, les traités de spiritualité rejoignent, dépassent même le sermon. En l'absence de toute poétique de ces textes, qui se définissent avant tout par l'ambition fondamentale de "traiter de $^{3}$ ", les traités sont implicitement relégués aux limbes du littéraire. Pourtant, quelques sondages dans l'immense production spirituelle du premier $\mathrm{xvI}^{\mathrm{e}}$ siècle espagnol permettent d'identifier des textes indéniablement traversés par une ambition esthétique, laquelle est le fruit d'une formation littéraire quelle qu'elle soit. Ainsi, en mettant en regard la prédication, pain quotidien des religieux ${ }^{4}$, et une production écrite plus libre et singulière, on peut tout à la fois envisager la pratique homilétique comme un atelier d'écriture et certains traités de spiritualité comme le produit de cette formation 
autodidacte pour une grande part. Pour les auteurs issus des ordres mendiants, rompus par conséquent à l'activité homilétique, un tel postulat paraît même s'imposer.

2 Le cas de Luis de Maluenda est exemplaire à cet égard. Ses traités donnent à voir des stratégies discursives et des procédés stylistiques divers issus sans conteste de la prédication et acclimatés à l'écriture spirituelle. Les récits profanes, les métaphores et comparaisons truculentes, la liberté de ton, la virulence de certaines formulations, le recours aux images en particulier, etc., sont à compter dans sa prose au nombre des expédients auxquels les prédicateurs recouraient pour capter l'attention de leur auditoire ${ }^{5}$. Ils lui confèrent des accents singuliers. Pourtant, aucun sermon de Maluenda ne nous est parvenu et ce qui intéresse sa connaissance de l'art de la prédication tient seulement de la présomption, si bien que se cache sous cette hypothèse de départ une pétition de principe qui conduit, comme il se doit, à la formulation d'une tautologie : l'allure supposément homilétique de la prose spirituelle de Maluenda est le reflet de l'influence du sermon sur l'écriture du traité. Prétendre explorer ainsi la nature du lien qui unit prédication et écriture spirituelle est, par conséquent, une impasse. Plus encore, c'est tenter de répondre à une question mal posée. En effet, il faut renoncer à rechercher, entre le sermon et le traité, une stricte relation d'inférence qui fait de celui-ci le reflet d'une performance réalisée dans un cadre contraint, pour les considérer sur un même plan, voire sur un pied d'égalité. Au reste, ce changement de perspective paraît s'imposer de façon naturelle chez des religieux pour lesquels la parole divine et son ministère s'inscrivaient dans une praxis si intimement liée à un type d'existence qu'elle en était à part égale la raison d'être et l'aboutissement.

3 Autrement dit, par sermon on ne désigne pas seulement une forme discursive s'inscrivant dans un contexte particulier, à la finalité catéchétique et réunissant les outils rhétoriques et les traits stylistiques propres à servir pareille ambition. En employant ce terme et dans la perspective retenue ici, il s'agit également de nommer un instrument qui permet d'observer la projection et l'expression littéraires d'un statut particulier, en l'espèce celui de frère mineur de la province de Saint-Jacques-deCompostelle et de sujet du royaume de Castille, et d'apprécier comment ils trouvent à s'articuler et à se manifester dans une société et un contexte donnés, l'Espagne de Charles Quint. À cet égard, les réflexions menées par Giorgio Agamben dans plusieurs ouvrages réunis en 2016 dans le volume intitulé Homo sacer ${ }^{6}$ s'avèrent remarquablement éclairantes. Tels que restitués dans leur dimension ontologique et anthropologique dans la pensée du philosophe italien, les concepts d'office et de liturgie ${ }^{7}$ permettent de saisir la tension entre vie consacrée et œuvre(s) sacrée(s), entre ministère et sacrement, toutes choses sur lesquelles la portée de l'œuvre spirituelle est elle-même indexée et qui trouvent dans le recours à l'image la quintessence de leur expression.

\section{Prédication et écriture spirituelle dans la Castille du premier $\mathrm{XVI}{ }^{\mathrm{e}}$ siècle}

On ignorerait tout de ce franciscain observant, et des œuvres dont il est l'auteur s'il n'avait disséminé quelques informations dans le prologue à son ultime traité, Leche de la fe del príncipe christiano, publié en $1545^{8}$. La récolte est bien maigre toutefois et le Poverello n'aurait sans doute pas renié cette humilité et ce geste de dépouillement presque absolu d'un membre de la famille séraphique. En revanche, la prose de 
Maluenda, qui vaut peut-être davantage que tous les éléments de biographie, laisse percevoir un caractère ombrageux, brosse en creux le portrait d'une personnalité non seulement préoccupée par les maux du temps et les tribulations des Castillans, mais prompte à braver les dangers et les interdits pour y remédier personnellement. En effet, les raisons ne manquaient pas à Luis de Maluenda tant pour se jeter corps et âme dans l'arène politique que pour aspirer à un renouveau de l'expérience spirituelle fondé sur la restauration des vertus de la vie religieuse et notamment monastique ${ }^{9}$. Son œuvre porte le souvenir vivace des désordres qui accompagnèrent l'arrivée de Charles Quint et des avides Flamands de sa suite en Espagne, peu avant l'élection impériale de 1519. Or les premiers porte-paroles et idéologues de cette révolution des Comunidades de Castille avaient été des religieux, augustins, dominicains et, bien évidemment, franciscains ${ }^{10}$. En prenant la plume pour sonner l'alarme devant certaines dérives du pouvoir royal, quelque vingt ans plus tard, Luis de Maluenda s'autorisait sans doute de la prédication de ces «moines frondeurs ${ }^{11}$ » de la fin des années 1510 et du début des années 1520. D'autre part, imprégné de la spiritualité du recogimiento, mouvement de renouveau de la foi et de la vie chrétienne issu de la Devotio moderna, Maluenda était naturellement sensible à la responsabilité qui incombait à l'institution ecclésiastique. En prônant l'union du corps et de l'âme, en revendiquant l'étroite combinaison d'exercices ascétiques et de méditations mystiques, les recogidos n'opposaient pas vie active et vie contemplative ${ }^{12}$. Or, depuis ses premières œuvres, Maluenda ne cessait d'observer et de dénoncer un dangereux déséquilibre à cet égard parmi ses contemporains ${ }^{13}$. De façon plus générale, dans une œuvre spirituelle qui ressortit au domaine de la théologie politique, le franciscain se lamente devant les conséquences funestes, en Castille et dans toute la chrétienté, d'un relâchement général du pouvoir politique et religieux.

5 On pourra objecter que les frères mineurs ou prêcheurs qui agitaient les foules au tournant des années 1510 étaient autrement exposés au danger que celui qui, arrivé au seuil de sa vie à n'en pas douter, rédige ses œuvres dans la tranquillité de sa cellule. Pourtant, l'intégration pleine et entière du politique dans le discours spirituel est sans doute de nature identique chez tous ces hommes. Il n'est pas indifférent qu'ils partagent une même audace, laquelle n'est selon toute vraisemblance que l'expression logique de l'impérieux devoir qui s'impose à eux. Or, la réalisation de la «forme-de$v^{1 e^{14}} »$ sur laquelle repose le monachisme contient en puissance les développements dont le dominicain Pablo de León, figure de proue des moines comuneros, ou Luis de Maluenda sont les représentants dans leur activité homilétique et scripturaire respectivement. Une fois encore, l'épisode des Comunidades, traumatique à maints égards pour les hommes de cette époque ${ }^{15}$, était propre à révéler la profondeur et la portée de cette forme-de-vie. Sommés de faire de l'éloquence sacrée une arme au service de la lutte contre un pouvoir dévoyé et impropre à garantir les intérêts des sujets et le salut des chrétiens, cette expérience avait montré à certains membres de communautés religieuses, s'il en était besoin, que la forma vivendi chrétienne, sans être étrangère à la sphère du droit, " ne peut se réduire à l'observance d'un précepte, ne peut avoir de nature légale ${ }^{16} »$. Un tel principe, central dans la vie monastique, trouvait son plus haut degré d'expression dans la famille du Poverello car, dans la tension instaurée par le franciscanisme entre règle et vie, l'application de la loi à la vie, selon le paradigme du pouvoir séculier et ecclésiastique, était dépassée ${ }^{17}$. 
6 À la vérité, nous ignorons pour une bonne part comment Maluenda, qui peut-être résidait au couvent des frères mineurs de Salamanque au plus fort de la prédication comunera, vécut les événements du tournant des années 1510. À en juger par le tempérament qui affleure à la surface de sa prose, le franciscain semble bien de la même trempe que ces prédicateurs qui firent de leur éloquence une arme et de leur chaire une forteresse, avant, pour les plus radicaux, de prendre d'autres armes. Au reste, un Miroir des franciscains de ces mêmes années ${ }^{18}$ apporte une série de précisions à propos des conditions d'exercice de la prédication qui placent le sermon au cœur de la dialectique entre règle/droit et vie. Hors des couvents franciscains ou des places royales, elle est soumise à l'autorisation du supérieur hiérarchique direct, curé, recteur, évêque, etc., et ne doit en aucun cas être le prétexte à la censure de cette autorité ou du pouvoir séculier ${ }^{19}$. Mais, s'empresse de préciser frère Gonzalo, compilateur de cette règle abrégée, Bernardin de Sienne avait déjà énoncé trois cas où cette prescription devait être suspendue: en cas de "péché manifeste, danger manifeste, risque de contagion manifeste ${ }^{20} »$. Il existait donc une marge d'appréciation qui était à la fois espace de liberté et de responsabilité, prudemment délimité par la menace de l'excommunication et du retrait définitif du droit de prêcher. À l'instar des prédicateurs, en qui il reconnait les premiers des martyrs ${ }^{21}$, Luis de Maluenda sait très bien quand il franchit les limites de ce qu'il est possible d'écrire dans la Castille des années 1530-1540. Il connaît donc les risques qu'il encourt et ne manque jamais une occasion d'y faire allusion, indiquant par là à son lecteur un passage de son discours à l'importance notoire, dans un geste qui donne à voir la prégnance de cette forme-de-vie monastique $^{22}$.

\section{Liturgie du texte spirituel}

7 À ce stade de la réflexion, le questionnement proposé en introduction à propos de la formation littéraire de ces auteurs spirituels mérite d'être reformulé. Si l'indifférenciation entre règle et vie est redoublée, à un niveau supérieur, par les similitudes entre prose des traités et discours homilétique, il faut chercher plus en amont encore, dans ce qui fonde la vie monastique et nourrit la forme-de-vie qui représente le stade ultime de son achèvement, la source de cette abolition des frontières entre les domaines existentiel et littéraire. À plus forte raison dans un contexte général de retour à l'observance favorisé par les Rois catholiques, autorisé par Alexandre VI et mis en œuvre par le cardinal Francisco Jiménez de Cisneros ${ }^{23}$. Dans la Castille du début du siècle, la restauration progressive de la discipline à l'intérieur des communautés faisait écho aux aspirations des moines tentés par la voie du recogimiento en rétablissant l'office divin au cœur de l'existence monastique. Ce faisant, tout en scandant la journée monastique, la lecture liturgique redevenait l'aliment essentiel de la spiritualité.

8 À la vérité, hormis la messe conventuelle quotidienne, et encore il n'y avait rien de systématique, les heures canoniques ne donnaient pas lieu à un sermon. Les liens entre prédication et liturgie n'en étaient pas moins étroits. Élément au statut longtemps incertain au sein de cette dernière, relais du texte sacré, les prêches devaient également être considérés comme inférieurs au texte des rites, dont les paroles étaient directement tirées des Écritures. Pour cette raison peut-être les ouvrages médiévaux où les offices sont décrits sont-ils particulièrement lapidaires au moment d'évoquer le 
sermon et la place qui doit lui être réservée ${ }^{24}$. Tout se passe comme s'il était naturel de statuer de manière privilégiée sur ce qui « exige une stabilité des formes d'expression, dans les paroles et dans les gestes", mais pas sur "la prédication [qui] tire son efficacité d'un ajustement plus circonstancié aux attentes, à la culture, aux expériences vécues de l'auditoire ${ }^{25}$ ». Pourtant, il y a un intérêt certain à discerner « les emprunts que font les prédicateurs à la liturgie, et même de saisir en quoi la démarche des prédicateurs révèle de leur part, tantôt une préoccupation de pastorale liturgique, et tantôt l'instrumentalisation de la liturgie au service des fins qui leur sont propres ${ }^{26}$ ».

Cette liturgie de la parole inscrite au cœur du quotidien trouve dans le dernier traité de Maluenda une modalité d'expression qui dépasse et transcende le cadre homilétique sans oblitérer la profondeur des liens entre sermon et liturgie et, de façon systématique, entre sermonnaires et année liturgique - et aux mystères dont elle fait mémoire ${ }^{27}$. Toutes proportions gardées pour des textes destinés à être lus, il semble bien que se joue dans les principaux choix stylistiques d'un traité tel que Leche de la fe del príncipe christiano l'instauration d'un rapport à la liturgie, voire la fondation d'une liturgie génératrice d'une performativité du discours qui introduit l'ouvrage sur un terrain analogue à celui occupé par le sermon. L'analyse micro et macro-structurelle de ce long texte permet d'isoler trois phénomènes particulièrement saillants. Par ordre croissant d'importance, il s'agit, d'un point de vue stylistique, de l'usage de l'hypotaxe ; sur le plan poétique, du choix du centon comme option générique fondamentale et, enfin, en termes rhétoriques, d'un soin particulier accordé à la dispositio.

Le premier de ces procédés est singulièrement représentatif d'une certaine prose castillane de la première moitié $d u \mathrm{xvI}^{\mathrm{e}}$ siècle chez Maluenda. Elle se déploie sur un mode accumulatif dans l'espace de ce qu'on ne parvient pas toujours à délimiter comme phrase ou période. En outre, le passage d'un chapitre à l'autre n'est guère accentué sur le plan typographique. Ainsi, le changement de caractère du titre, lui-même précédé d'un pied-de-mouche annonciateur d'un nouveau paragraphe, est salutairement renforcé par la présence d'une majuscule de grande taille et historiée. Le traité dans son ensemble et ses longs chapitres en particulier se présentent donc sous la forme d'un continuum syntaxique.

11 Ce principe élémentaire est également un procédé qui permet, à un second niveau, de tisser un maillage extrêmement subtil entre le propos de l'auteur du traité et les citations aussi diversifiées que nombreuses qui le traversent ou entre lesquelles il vient se nicher. Il n'est pas indifférent, à cet égard, que Leche de la fe del príncipe christiano présente pour une part importante la physionomie du centon. Il relève autrement dit d'un des cadres génériques où l'auctorialité est réduite à sa plus simple expression, quand elle n'a pas vocation à disparaître entièrement. Par ce choix, Maluenda compense, sans doute de façon inconsciente, le défaut d'humilité causé par les révélations autobiographiques du prologue. Tout en y reprenant pour lui-même la métaphore du canal, comme image des clercs ou des prédicateurs, médiateurs entre Dieu et les hommes, qui font couler dans leur propre discours la parole de Dieu... il s'était empressé d'ajouter toutefois qu'il était bien sec pour sa part ${ }^{28}$. Cette justification, suspecte de répondre à une stratégie de captatio benevolentice, n'est pas incompatible avec une conception du texte spirituel comme espace discursif où Verbe de Dieu, commentaires sacrés et parole de celui qui le déclare sont indissociables ${ }^{29}$. À cet égard, la composition du centon est similaire à celle du sermon, du moins sous sa forme latine, qu'Hervé Martin décrit comme «quasi mécanique, par assemblage d'énoncés 
préexistants, sur le mode d'une sorte de lego ${ }^{30} »$. De fait, à l'autre bout du siècle et dans un contexte différent à maints égards, Juste Lipse dans le De consilio et forma nostri operis revendiquait cette forme où « tout est nôtre, et [où] rien ne l'est ${ }^{31}$ ». Maluenda aurait pu souscrire à cette maxime, non pas dans un geste d'appropriation mais plutôt avec la volonté de se consacrer lui-même comme ministre de la parole, officiant - en sa qualité d'ordonnateur d'une liturgie textuelle - et prédicateur à la fois.

En dernière analyse, il n'est donc pas indifférent que la dispositio des chapitres du traité, qui répondent, à l'exception des trois premiers, à une même division tripartite, soit évocatrice d'une économie liturgique de ces mêmes chapitres. Dans leur première partie, Maluenda expose les vertus du prince chrétien ou les vices de l'Antéchrist, autant d'indices destinés à permettre à ses contemporains de distinguer l'un de l'autre. Entre les nombreuses autorités - depuis les philosophes païens jusqu'aux Pères et théologiens de tous temps, en passant par les Écritures - vient parfois se nicher le discours critique de Maluenda. Avec une grande liberté de ton, le franciscain dénonce, çà et là, les excès et les dérives du pouvoir royal et ecclésiastique. La seconde et la troisième parties suivent, en revanche, un invariable patron: après l'exposition d'un miracle ou d'un mystère de la vie du Christ ou de la Vierge, le chapitre se referme inexorablement sur la présentation d'une ou deux figurations de l'Antéchrist à travers l'histoire. Le traité observe ainsi une progression chronologique, qui est aussi, pour la partie centrale, celle de l'organisation de l'année liturgique, depuis l'Incarnation jusqu'à l'Assomption de la Vierge, tandis que l'évocation de l'Antéchrist conduit le lecteur de Caïn et Simon le Mage, au chapitre IV, jusqu'à Alexandre et Mahomet dans le soixante-quatrième ${ }^{32}$. Ainsi, en vertu de sa position centrale, la composante homilétique - au sens précis de cette épithète, en tant qu'appliquée au commentaire d'une péricope - agit comme un pivot autour duquel s'articule l'intégralité du propos de chacun des chapitres, sans aucune solution de continuité à l'intérieur de cette économie interne, la dernière phrase de la première section devenant la première de celle-ci, et ainsi de suite entre cette dernière et la partie finale consacrée à l'Antéchrist. En somme, cet usage de la dispositio, qui fait fond sur le recours à l'hypotaxe et s'inscrit de plain-pied dans la poétique du centon, qui est à certains égards celle du sermon également, engendre un dispositif textuel qui soumet le lecteur à une forme de lectio continua, pain quotidien de la vie monastique et renouveau de la vie spirituelle. À travers cette articulation des trois principaux niveaux de l'économie du traité, Maluenda semble consacrer la pastorale évangélique comme le fondement de Leche de la fe del príncipe christiano. Tout se passe donc comme si ce qui est à la fois le socle de l'office du prédicateur et le cœur d'une liturgie monastique de la parole devenait le principe structurant d'une liturgie textuelle dont l'auteur du traité est, à plus d'un titre, l'officiant.

\section{Office des images spirituelles}

13 Dans Leche de la fe, le franciscain Luis de Maluenda propose donc à son lecteur de prendre part à une liturgie du texte spirituel qui, par une ritualisation du discours, donne à voir les mystères du salut à un rythme dont la régularité presque impeccable est assurée par l'économie simple et efficace du traité. Ce faisant, il convie ce même lecteur - qui n'est autre peut-être que son frère en religion - à pratiquer une lecture liturgique qui met en tension les événements divers de l'histoire du salut et ceux du 
temps messianique. Dans l'exploration des liens entre sermon et traité de spiritualité, on peut s'interroger, dans un tel dispositif, sur le rôle joué par les images dont le texte regorge. Giorgio Agamben, dans De la très haute pauvreté, cite un passage de l'Explication de la divine liturgie de Nicolas Cabasilas, théologien byzantin du $\mathrm{XIV}^{\mathrm{e}}$ siècle, remarquablement éclairant quant à l'articulation entre performativité du discours spirituel et dimension visuelle des offices liturgiques :

Dans les paroles lues ou chantées, «nous voyons (horōmen) la représentation du Christ, des œuvres qu'il a accomplies et des souffrances qu'il a endurées pour nous. Dans les psalmodies et les lectures, comme dans tous les actes du prêtre à travers l'ensemble des rites, est signifiée (sèmainetai) toute l'économie du Sauveur ». Et « si la mystagogie tout entière est comme l'icône d'un corps qui est la vie du sauveur ", les chants et les lectures signifient et nous «mettent sous les yeux (hyp'opsin agousa) les différents moments de l'économie du Christ ${ }^{33}$.

Dans Leche de la fe del príncipe christiano, la présence du visible atteint un degré de saturation générale ${ }^{34}$ qui est une première marque de l'étroitesse des liens qui unissent la prose des traités et la pratique homilétique. Mais peut-être les grandes qualités visuelles de certaines descriptions des miracles ou simples scènes de la vie du Christ qui émaillent le traité de Maluenda sont-elles les marques les plus éclatantes de cette communauté littéraire. La manière dont Maluenda traite le Lavement des pieds est exemplaire à cet égard. Avec un luxe de détails pratiques, il convie le lecteur à se représenter Jésus agenouillé, une serviette nouée à la ceinture, les manches de sa tunique relevées et jusqu'à ses cheveux retenus derrière les oreilles pour mieux donner à voir, bien évidemment, que le Christ se penche sur les pieds d'un apôtre ${ }^{35} . .$. toutes choses que le prédicateur aurait pu mettre en œuvre si son sermon avait porté sur Jean 13,1-17, où est rapporté le récit du Lavement des pieds. Quoique sur un mode différent, les observations de Nicolas Cabasilas sont aisément applicables au personnage et aux actions de l'Antéchrist, autre grand catalyseur des effets visuels dans la littérature qui lui est consacrée ${ }^{36}$. En effet, le discours messianique comporte en luimême tout un arsenal d'images plus saisissantes les unes que les autres au sujet de ce personnage au rôle important dans l'économie du salut. Il n'est donc pas indifférent que la troisième section de chacun des chapitres propose expressément des portraits de cette bête de l'Apocalypse dont les innombrables têtes portent les traits de ces hommes qui, par leur comportement, préfigurèrent l'arrivée de la fin des temps.

De façon générale, le discours de Maluenda, dans l'ensemble du traité, est traversé par les champs sémantiques de la vision et de la représentation. Ils ressortissent, en premier lieu, à la dimension allégorique de Leche de la $f e$, ouvrage qui puise abondamment dans les trésors de ce type d'exégèse. Le verbe figurar et ses dérivés sont amplement représentés. Pintar l'est peut-être encore davantage ${ }^{37}$. Quoique les dictionnaires de l'époque moderne n'attestent pas le sens figuré de pintar - il n'est enregistré pour la première fois que dans le Diccionario de Autoridades durant la première moitié $d u$ XvIIII $^{\mathrm{e}}$ siècle - , les auteurs spirituels de l'époque de Maluenda paraissent user de ce vocable avec naturel pour désigner la représentation mentale d'une scène ou d'une réalité concrète, voire incorporelle ${ }^{38}$. Maluenda ne déroge pas à cette règle, si bien qu'Apelle en personne ${ }^{39}$ est dépassé en virtuosité par saint Bernard. Sa peinture des pains des sept miséricordes surpasse une représentation aussi subtile que celle des couleurs de la brume, voire des affects et des passions du cœur, tel que Maluenda le précise après une longue citation du troisième sermon pour le sixième dimanche après la Pentecôte ${ }^{40}$. En comparant le parangon antique du peintre et le 
docteur melliflue, insigne prédicateur de la chrétienté médiévale et tout spécialement de la deuxième croisade, le franciscain révèle, sur la question particulière des qualités visuelles de sa prose, ce que cette dernière doit à l'art du sermon. En inscrivant vertus et artifices du sermon au cœur des chapitres de Leche de la fe del príncipe christiano, il lui confère une capacité particulière à mettre sous les yeux du lecteur ce que l'accomplissement des rites durant les célébrations liturgiques donnait à voir par d'autres moyens. Le cas du Lavement des pieds est exemplaire à cet égard.

À travers ce rapprochement avec saint Bernard, dans l'œuvre duquel Luis de Maluenda puise abondamment, on perçoit sans doute le fondement même de la chaîne qui paraît unir le sermon en général et le traité Leche de la fe del príncipe christiano en particulier, et qui passe notamment par le recours à des effets visuels qui rendent la prose remarquablement plastique. En effet, en mettant un tel soin, sur une œuvre remarquablement longue, à déployer une économie liturgique du texte spirituel, le franciscain remplit étymologiquement l'office du leitourgos, autrement dit de celui qui est chargé d'une mission communautaire, qui apporte une prestation publique et sacrificielle. Au reste, l'Épitre aux Hébreux substitue au terme liturgie celui d'office lorsque cette mission est d'ordre spécifiquement politique ${ }^{41}$. L'activité de Bernard de Clairvaux, à l'intérieur de son ordre et dans le siècle, fournissait un insigne exemple à cet égard à Luis de Maluenda.

17 L'engagement spirituel et politique de ce dernier sur les grandes questions qui agitent la Castille de son époque passe bien par l'exhibition d'un diptyque dont les deux volets donnent à voir, en regard les uns des autres, les mystères de l'année liturgique et les doubles portraits de l'Antéchrist - princes temporels et hérésiarques - à travers l'histoire des hommes. De même que la lecture liturgique et son commentaire homilétique mettent sous les yeux des fidèles les épisodes de la vie du Sauveur et, par conséquent, l'économie de la rédemption, de même le discours spirituel doit-il donner à voir, au chrétien ou au moine qui lisent dans l'intimité de leur foyer ou de leur cellule, tout un office textuel qui est fondamentalement forme de vie et au cœur duquel se trouvent également inscrits l'essentiel du message eschatologique et la batterie d'effets visuels qu'il véhicule. Ainsi, le ministère de la parole est intrinsèquement un ministère de l'image. Transposé dans l'espace de Leche de la fe del príncipe christiano, ces deux discours complémentaires s'inscrivent dans une liturgie textuelle fondatrice d'une économie littéraire et qui consacre l'auteur en officiant. Son œuvre cesse ainsi d'être le fruit de sa seule vertu pour devenir le lieu d'expression du mystère du salut et de la parousie, dont il n'est que l'instrument et qu'il donne à voir autant qu'il le traduit verbalement. Par sa régularité, la rigoureuse économie du traité révèle le fondement de la mission pastorale du prédicateur, explication des mystères et admonestations morales; le transfert du recours aux images, comme artifice de choix, depuis le sermon jusqu'à la prose spirituelle trouve, de ce point de vue, une explication particulière. En outre, ce dispositif invite le lecteur à prendre part à un office assumé par l'auteur du traité dans un discours émaillé des effets de l'éloquence sacrée, au premier rang desquels il convient de placer le recours au visuel. À travers ce geste, qui tend vers la conciliation de la performativité de la parole et de l'effectualitéte de l'action liturgique, l'officiant ne donne rien moins à voir que son propre sacrifice, conformément à la forme-de-vie. 


\section{BIBLIOGRAPHIE}

AgAmBEN Giorgio, Homo sacer. L'intégrale : 1997-2015, M. Rosa (trad.), Paris, Seuil, 2016.

AgAmBEn Giorgio, Opus Dei. Archéologie de l'office, M. Rueff (trad.), dans Id., Homo sacer. L'intégrale 1997-2015, Paris, Seuil, 2016, p. 689-810.

Agamben Giorgio, De la très haute pauvreté, dans Id., Homo sacer. L'intégrale : 1997-2015, M. Rosa (trad.), Paris, Seuil, 2016, p. 939-1051.

ALBA Ramón, Acerca de algunas particularidades de las Comunidades de Castilla tal vez relacionadas con el supuesto acaecer terreno del Milenio Igualitario, Madrid, Editora Nacional, 1975.

ANDRÉs MARTín Melquíades, Los recogidos. Nueva vision de la mística Española (1500-1700), Madrid, Fundación Universitaria Española, 1976.

BÉRIou Nicole et Morenzoni Franco, Prédication et liturgie au Moyen Âge, Turnhout, Brepols, 2008.

ChaRAnsonnet Alexis, « Traces de mises en scène spectaculaires de la parole du prédicateur. Trois sermons d'Eudes de Châteauroux aux Parisiens (années 1229-1230) ", dans M. Bouhaïk-Gironès et M. A. Polo de Beaulieu (dir.), Prédication et performance du XII au XIII ${ }^{e}$ siècle, Paris, Garnier, 2013, p. 19-45.

DELCORNO Carlo, « Liturgie et art de bien prêcher (XIII ${ }^{\mathrm{e}}-\mathrm{XV}^{\mathrm{e}}$ siècle) », dans N. Bériou et F. Morenzoni (dir.), Prédication et liturgie au Moyen Âge, Turnhout, Brepols, 2008, p. 201-221.

GILBERT Françoise, La figure de l'Antéchrist dans l'Espagne du Siècle d'or, thèse de doctorat de l'université Toulouse 2-Le Mirail, sous la direction de Marc Vitse, 1995.

GoNZALo Fray, Espejo de frailes menores, Séville, Juan Cronberger, 1531.

GuAdalajaRa Medina José, El Antichristo en la España medieval, Madrid, Ediciones del Laberinto, 2004.

INFANTES Víctor, «Tipologías de la enunciación literaria en la prosa áurea. Seis títulos (y algunos más) en busca de un género: obra, libro, tratado, crónica, historia, cuento, etc. (IV) », dans F. Sevilla Arroyo et C. Alvar Ezquerra (dir.), Actas del XIII congreso de la Asociación Internacional de Hispanistas (Madrid, 6-11 juillet 1998), Madrid, Castalia, 2000, vol. 3, p. 641-654.

LAFOND Jean, « Le centon et son usage dans la littérature morale et politique », dans J. Lafond et A. Stegmann (dir.), L'automne de la Renaissance 1580-1630, Paris, Librairie philosophique J. Vrin, 1981, p. 118-128.

LONGÈRE Jean, La prédication médiévale, Paris, Études augustiniennes, 1983.

MALUENDA Luis DE, Tratado llamado leche de la fe del príncipe christiano. Con LXII milagros de Jesu christo nuestro Dios y redemptor. $Y$ con los misterios del antechristo: $Y$ con las ropas de las virtudes Morales $y$ Theologales. Copilado de los excelentes libros por fray Luys de Maluenda. A los quarenta años de ávito de religioso menor. Y examinado por el Reverendíssimo Padre ministro general: y aprovado por el Consejo Real, Burgos, Juan de Junta, 1545.

MARTIN Hervé, Le métier de prédicateur à la fin du Moyen Âge, 1350-1520, Paris, Cerf, 1988.

OsunA Francisco DE, Segunda parte del Abecedario espiritual, J. J. Morillo Pérez (éd.), Madrid, Cisneros, 2004 (1530). 
OsUNA Francisco DE, Quinta parte del Abecedario espiritual, M. Quirós García (éd.), Madrid, Fundación Universitaria Española, 2002 (1540).

PÉREZ Joseph, « Moines frondeurs et sermons subversifs en Castille pendant le premier séjour de Charles Quint en Espagne », Bulletin hispanique, t. 67, n 1-2, 1965, p. 5-24.

PÉREZ Joseph, La révolution des « Comunidades » de Castille (1520-1521), Bordeaux, Féret \& Fils éditeurs, 1970.

QUERO Fabrice, « Expérience individuelle, littéraire et politique dans l'œuvre de Luis de Maluenda : le $221^{\mathrm{e}}$ adage d'Érasme et le $5^{\mathrm{e}}$ chapitre de Leche de la fe del príncipe christiano ", eSpania, $\mathrm{n}^{\circ}$ 25, 2016, URL : <http://journals.openedition.org/e-spania/26149>.

QUERO Fabrice, « Le visible saturé du Tratado llamado leche de la fe del príncipe christiano de Luis de Maluenda (1545) ", L'Âge d'or, nº 5, 2012, URL : <http://journals.openedition.org/agedor/1011>.

\section{NOTES}

1. Face à Michel Zink qui parlait de «sermon dans un fauteuil » pour la production homilétique consignée par écrit, on peut suivre A. Charansonnet, « Traces de mises en scène spectaculaires de la parole du prédicateur. Trois sermons d'Eudes de Châteauroux aux Parisiens (années 1229-1230) ", dans M. Bouhaïk-Gironès et M. A. Polo de Beaulieu (dir.), Prédication et performance $d u X^{e} I^{e}$ au XIII siècle, Paris, Garnier, 2013, p. 19-45, qui décèle diverses traces de la performance orale dans le corpus qu'il étudie.

2. Sur ce point, on se référera de façon générale à l'ouvrage classique de J. Longère, La prédication médiévale, Paris, Études augustiniennes, 1983, p. 177-202 notamment.

3. V. Infantes, «Tipologías de la enunciación literaria en la prosa áurea. Seis títulos (y algunos más) en busca de un género: obra, libro, tratado, crónica, historia, cuento, etc. (IV)», dans F. Sevilla Arroyo et C. Alvar Ezquerra (dir.), Actas del XIII congreso de la Asociación Internacional de Hispanistas (Madrid, 6-11 juillet 1998), Madrid, Castalia, 2000, vol. 3, p. 641-654.

4. Comme le rappelle Hervé Martin dans Le métier de prédicateur à la fin du Moyen Âge, 1350-1520, Paris, Cerf, 1988, p. 115 : « On connaît l'adage qui résume les études en théologie : legere, disputare, prcedicare. Pour certains étudiants avancés, comme les bacheliers des couvents de mendiants, c'est un simple complément de formation puisqu'ils prêchent déjà aux foules, après y avoir été initiés au sein de leur ordre. » Nicole Bériou, dans son introduction à l'ouvrage coordonné avec Franco Morenzoni, Prédication et liturgie au Moyen Âge, Turnhout, Brepols, 2008, p. 10-11, ajoute que c'était là une obligation pour tout clerc.

5. Voir J. Longère, ouvr. cité, p. 228-230.

6. G. Agamben, Homo sacer. L'intégrale: 1997-2015, M. Rosa (trad.), Paris, Seuil, 2016.

7. Nous nous référons en particulier à De la très haute pauvreté. Règles et forme de vie, J. Gayraud (trad.), ibid., p. 943-1050 (2011).

8. L. de Maluenda, Tratado llamado leche de la fe del príncipe christiano. Con LXII milagros de Jesu christo nuestro Dios y redemptor. Y con los misterios del antechristo: $Y$ con las ropas de las virtudes Morales y Theologales. Copilado de los excelentes libros por fray Luys de Maluenda. A los quarenta años de ávito de religioso menor. Y examinado por el Reverendíssimo Padre ministro general: y aprovado por el Consejo Real, Burgos, Juan de Junta, 1545. Sur la biographie de ce personnage, nous nous permettons de renvoyer à F. Quero, "Expérience individuelle, littéraire et politique dans l'œuvre de Luis de Maluenda : le $221^{\mathrm{e}}$ adage d'Érasme et le $5^{\mathrm{e}}$ chapitre de Leche de la fe del príncipe christiano ", eSpania, $\mathrm{n}^{\circ}$ 25, 2016, URL : <http://journals.openedition.org/e-spania/26149>.

9. Dans Leche de la fe, ouvr. cité, f. ccXlvi, n'affirme-t-il pas que les monastères sont le séjour de la plupart des démons car ils y sont offensés par les occupations vertueuses de leurs habitants? 
10. J. Pérez, dans La révolution des "Comunidades" de Castille (1520-1521), Bordeaux, Féret \& Fils éditeurs, 1970, p.503, allait même jusqu'à préciser: "Entre franciscains et comuneros, les rapports semblent toujours avoir été très cordiaux. »

11. L'expression appartient à J. Pérez, «Moines frondeurs et sermons subversifs en Castille pendant le premier séjour de Charles Quint en Espagne ", Bulletin hispanique, t. 67, nº 1-2, 1965, p. 5-24.

12. M. Andrés Martín, Los recogidos. Nueva vision de la mística Española (1500-1700), Madrid, Fundación Universitaria Española, 1976, p. 13.

13. M. Avilés Fernández, «El anónimo franciscano de la provincia de Santiago », dans M. Andrés Martín, Los recogidos, ouvr. cité, p. 256.

14. G. Agamben, De la très haute pauvreté, ouvr. cité, p. 9, définit ainsi cette notion : «Une vie si étroitement liée à sa forme qu'elle s'en montre inséparable. »

15. R. Alba, Acerca de algunas particularidades de las Comunidades de Castilla tal vez relacionadas con el supuesto acaecer terreno del Milenio Igualitario, Madrid, Editora Nacional, 1975, p. 83-94.

16. G. Agamben, De la très haute pauvreté, ouvr. cité, p. 66.

17. Ibid., p. 126.

18. F. Gonzalo, Espejo de frailes menores, Séville, Juan Cronberger, 1531.

19. Ibid., f. $x x x v v^{\circ}$.

20. Ibid., f. XXXIIII $\mathrm{r}^{\circ}$.

21. Cette précision apparaît au détour du portrait idéal du prédicateur brossé par Maluenda au chapitre XLI de Leche de la fe, ouvr. cité, f. cCLXXIIII $\mathrm{v}^{\circ}:$ " Y el primero linaje y género de martirio es el martirio de la predicación evangélica porque el predicador, que en su predicación es limpio de mentiras contra verdad de las sanctas escripturas, para lo cual son menester grandes trabajos y cuidados y en su vida y obras, es ajeno de vicios que contradicen a la doctrina que predica, y es predicador universal de las verdades generales de los pueblos y de las repúblicas y sin ningún interese temporal; a este tal, por mártir le han de contar porque este estilo de predicación es un martirio estrechísimo que testifica la verdad evangélica con sudores de sangre. "

22. Il est remarquable que Maluenda recoure souvent à des formules conclusives de prétérition du type «o sino, yo callo por el tiempo en que me hallo». Ainsi, lorsqu'il dénonce, à travers la métaphore de la vente aux enchères, la simonie qui affectera toutes les charges ecclésiastiques à la fin des temps: "Así andarán en aquellos tiempos finales los pontificados y dignidades de la Iglesia puestas en almoneda de dineros, que quien más dinero diere que sea suyo el sacerdocio y obispado, y el oficio de la Iglesia. Y junto con esto el sacerdocio estará mudo porque los justos y los sabios sacerdotes y obispos estarán mudos y no osarán hablar contra los vicios y maldades de las repúblicas sino a grande costa de persecuciones conforme a lo que dize el profeta: "Son perros mudos que no pueden ni osan ladrar." Y si algunas centellas destas pestilencias destas almonedas destos oficios y beneficios eclesiásticos han començado a volar por el estado de la Iglesia, o no, yo callo por el tiempo en que me hallo, y callando yo, escriba aquí la pluma las palabras que dixo Salustio de la ciudad de Roma. " (L. de Maluenda, Leche de la fe del príncipe christiano, ouvr. cité, f. LXIX $\mathrm{r}^{\mathrm{o}}$, ou encore f. $\mathrm{CXC}^{\circ}$.)

23. M. Andrés Martín, Los recogidos, ouvr. cité, p. 26.

24. Précision apportée par N. Bériou, en introduction à N. Bériou et F. Morenzoni, Prédication et liturgie au Moyen Âge, ouvr. cité, p. 10.

25. Ibid.

26. Ibid., p. 16.

27. Sur cet aspect du sermon, on pourra se reporter, notamment, au début du chapitre de C. Delcorno, "Liturgie et art de bien prêcher ( $\mathrm{XIII}^{\mathrm{e}}-\mathrm{XV}^{\mathrm{e}}$ siècle) ", dans N. Bériou et F. Morenzoni, Prédication et liturgie au Moyen Âge, ouvr. cité, p. 201-221.

28. L. de Maluenda, Leche de la fe del príncipe christiano, ouvr. cité, f. AvIII $\mathrm{r}^{\circ}$. 
29. Voici un passage où Maluenda mêle étroitement des considérations contemporaines qui relèvent de son propre discours à celles de saint Jérôme. Ce faisant, il fustige les dejados, ces partisans d'une lecture radicale de la spiritualité illuministe. En s'abandonnant à la grâce divine, ils prétendaient, en effet, accéder à un état d'impeccabilité : "[...] aquel erege pelagiano llamado Juliano y obispo de Capua en los tiempos de una grande hambre con las rentas de su obispado gastando largamente, ganó a muchos para que siguiesen la opinión herrada de su seta pelagiana ablandados con el estiércol de Flandes y con la manteca de los veneros de las indias según refiere san Jerónimo. E como en estos reynos los capitanes y las capitanas de las setas de dexados y de los beatos, tenían grandes gastos para los de sus setas, que para esto del demonio sembrador de bruxas les negociava abastanza de dineros, porque jamás uvo bruxo ni bruxa pobres [...]. » (Ibid., f. cCLXIII ; c'est nous qui soulignons.)

30. H. Martin, ouvr. cité, p. 255.

31. Cité par J. Lafond, «Le centon et son usage dans la littérature morale et politique », dans J. Lafond et A. Stegmann (dir.), L'automne de la Renaissance 1580-1630, Paris, Librairie philosophique J. Vrin, 1981, p. 118.

32. Encore faut-il préciser qu'Alexandre est déjà apparu comme préfiguration de l'Antéchrist au chapitre XXv, tout comme Hadrien, à qui il est associé au même endroit, apparaît une nouvelle fois au chapitre LXV. En réalité, la présentation chronologique s'achève au chapitre LXIII avec l'empereur Louis IV.

33. G. Agamben, De la très haute pauvreté, ouvr. cité, p. 100.

34. Sur cet aspect en particulier, voir F. Quero, « Le visible saturé du Tratado llamado leche de la fe del príncipe christiano de Luis de Maluenda (1545)», L'Âge d'or, nº 5, 2012, URL: <http:// journals.openedition.org/agedor/1011>.

35. L. de Maluenda, Leche de la fe del príncipe christiano, ouvr. cité, chap. XL, f. CCLXV : «Y también en el Jueves sancto milagroso obró el Rey de gloria aquella hazaña milagrosa que vale por cien mil milagros, la cual fue el Lavatorio de pies que el Redemptor con aquellas sus manos virginales y divinas, hincado de rodillas, y ceñido una tobaja, las mangas de la túnica levantadas hasta los codos sagrados, los cabellos nazareos recogidos detrás de sus sagradas orejas, con inefable fervor de caritativa humildad y diligencia, lavaba los pies de los pobres discípulos [...]. »

36. Voir J.Guadalajara Medina, El Antichristo en la España medieval, Madrid, Ediciones del Laberinto, 2004, ou encore F. Gilbert, La figure de l'Antéchrist dans l'Espagne du Siècle d'or, thèse de doctorat de l'université Toulouse 2-Le Mirail, sous la direction de Marc Vitse, 1995.

37. Sur ces deux points en particulier, il serait nécessaire d'introduire ici des données statistiques précises que nous ne sommes malheureusement pas en mesure de fournir. À partir de quelques sondages effectués çà et là, il est permis de préciser que les emplois de figurar ne ressortissent pas uniquement au domaine de l'exégèse typologique. À titre d'exemple : «Y por las çarças moras que son manjar de pastores, son figuradas las dotrinas, y ceremonias, $y$ historias, y figuras de la ley vieja, las cuales son muy santas y muy buenas en su estilo y manera para los que gobiernan repúblicas y reinos [...]. » (Leche de la fe del príncipe christiano, ouvr. cité, prologue, f. AIIII $\mathrm{r}^{\circ}$.)

38. Voici, à titre d'exemple, deux passages tirés de l'œuvre d'un maître de la spiritualité franciscaine du recogimiento, Francisco de Osuna. Aux pages 427-428 de la Quinta parte del Abecedario espiritual, M. Quirós García (éd.), Madrid, Fundación Universitaria Española, 2002 (1540), on peut lire un passage où le franciscain use d'une double comparaison pour figurer l'inconstance humaine face à l'inébranlable engagement du Christ. Il rapporte tout d'abord l'histoire d'une vieille femme qui vendait des draps sales mais sur lesquels, sans les avoir lavés, elle avait pris le soin de peindre les portraits des douze pairs de France pour en augmenter le prix et augmenter la dot de sa fille. Il ajoute ensuite que la relation du chrétien au Sauveur est semblable à une conversation interrompue, intermittente. Ainsi : "Si sobre estas roturas quieres pintar los hechos apostólicos, que son obediencia y pobreza y castidad, primero as de sanear muy de espacio las quiebras passadas y restaurar muy bien la guarda de los mandamientos, para que, según lo sobredicho, te ame nuestro Señor.» Dans la Segunda parte del Abecedario espiritual, J. J. Morillo Pérez (éd.), 
Madrid, Cisneros, 2004, p. cIV (1530), on trouve par ailleurs : « Según dize un doctor, todas las cosas acabó Dios en seys días, y al hombre se dexó en alguna manera imperfecto y por acabar y como medio hecho. Y diole poder que se acabasse él de hazer, y se pintasse y debuxasse al talle y medida que por bien tuviesse dexándolo a la mano de su propio consejo, quedando Dios quasi juramentado para le ayudar concurriendo con él para se pintar y conformar a lo que por bien tuviesse tomando la semejança que más le agradasse, de manera que sea el hombre pintor de símesmo. »

39. « Ni Apeles el gran pintor, ni los otros primos pintores pudieron pintar más sutilmente los vientos que pintaban, como sant Bernardo pintó a los preñados de dinero. » (Leche de la fe, ouvr. cité, f. xxxvi vo et LXXVI $v^{\circ}$.)

40. "¿Qué pintor huvo en el mundo que fuese tan primo que pintase los colores de las nieblas que son muy dificultosas de pintar y que pintase los colores de los afectos y pasiones del corazón tan al propio, como el devotísimo doctor sant Bernardo pintó los panes de las siete misericordias? » (Ibid., f. CcXcVII $\mathrm{r}^{\circ}$ )

41. G. Agamben, Opus Dei. Archéologie de l'office, M. Rueff (trad.), dans Id., Homo sacer, ouvr. cité, p. 701 et 710 .

42. G. Agamben (ibid., p. 736) forge ce néologisme à partir des réflexions d'Odon Casel sur le terme effectus, qui traduit la marque de l'image et de la présence sacrée dans le mystère liturgique.

\section{RÉSUMÉS}

Afin d'approcher la singularité de la prose et du discours spirituels de Luis de Maluenda dans son dernier traité, cet article interroge les liens entre pratique de la prédication et écriture spirituelle en les replaçant dans une dynamique qui est propre à la forme-de-vie franciscaine, selon le sens que Giorgio Agamben donne à cette notion, et à la praxis qu'elle appelle. En mettant au jour l'existence d'une véritable liturgie textuelle à l'œuvre dans Leche de la fe del príncipe christiano, il s'agit de restituer à l'importante composante visuelle de ce texte sa fonction au sein d'un tel dispositif et de montrer ce qu'elle doit au sermon.

Allo scopo di evidenziare la singolarità della prosa e del discorso spirituale di Luis de Maluenda nel suo ultimo trattato, questo articolo esamina le connessioni tra la pratica della predicazione e la scrittura spirituale, esaminandole nella dinamica propria della forme-de-vie francescana (secondo il significato attribuito a questa nozione da Giorgio Agamben) e della prassi che essa chiama. Evidenziando l'esistenza di una vera liturgia testuale in Leche de la fe del príncipe christiano, si tratta di restituire all'importante componente visiva di questo testo la sua funzione all'interno di tale dispositivo e di mostrare ciò che esso deve alla predica.

In order to better apprehend the singularity of Luis de Malenda's prose and spiritual discourse in his last treatise, this article looks at the connections between preaching and spiritual writing within the perspective of a Franciscan forme-de-vie, in Giorgio Agamben's terms, and the praxis it calls for. The textual liturgy at work in Leche de la fe del príncipe christiano is linked to an important visual quality owing much to sermons. 
INDEX

Parole chiave : prediche, trattati spirituali, liturgia, immagini, Luis de Maluenda, Spagna,

XV secolo

Mots-clés : sermon, traités de spiritualité, liturgie, images, Luis de Maluenda, Espagne,

XVIe siècle

Keywords : sermons, spiritual treatises, liturgy, images, Luis de Maluenda, Spain, 16th Century

\section{AUTEUR}

\section{FABRICE QUERO}

Université Paul Valéry Montpellier 3, LLACS EA 4582.

fabrice.quero@univ-montp3.fr 